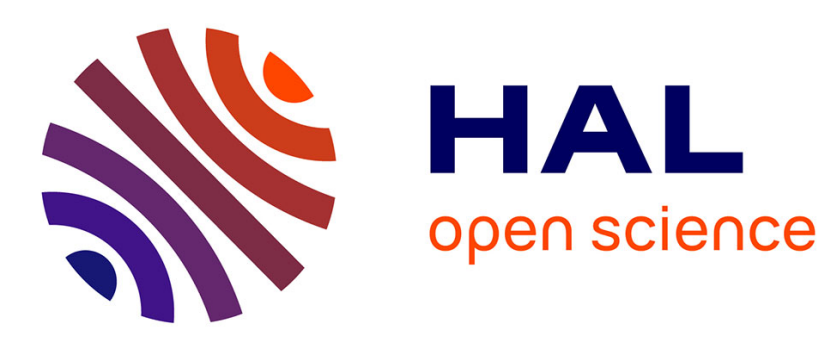

\title{
Electric Field Induced Lyman- $\alpha$ Emission of a hydrogen beam for electric field measurements
}

Laurence Chérigier-Kovacic, Peter Ström, Aurélien Lejeune, F Doveil

\section{To cite this version:}

Laurence Chérigier-Kovacic, Peter Ström, Aurélien Lejeune, F Doveil. Electric Field Induced Lyman- $\alpha$ Emission of a hydrogen beam for electric field measurements. Review of Scientific Instruments, 2015, 86 (6), pp.063504. 10.1063/1.4922856 . hal-01466150

\section{HAL Id: hal-01466150 https://hal-amu.archives-ouvertes.fr/hal-01466150}

Submitted on 13 Feb 2017

HAL is a multi-disciplinary open access archive for the deposit and dissemination of scientific research documents, whether they are published or not. The documents may come from teaching and research institutions in France or abroad, or from public or private research centers.
L'archive ouverte pluridisciplinaire HAL, est destinée au dépôt et à la diffusion de documents scientifiques de niveau recherche, publiés ou non, émanant des établissements d'enseignement et de recherche français ou étrangers, des laboratoires publics ou privés. 


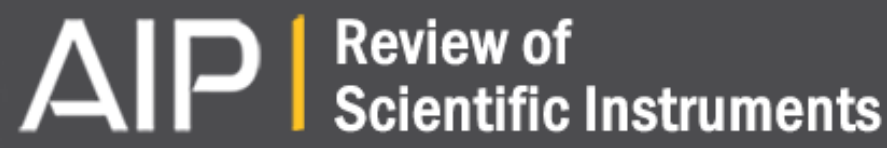

\section{Electric field induced Lyman-a emission of a hydrogen beam for electric field measurements}

L. Chérigier-Kovacic, P. Ström, A. Lejeune, and F. Doveil

Citation: Review of Scientific Instruments 86, 063504 (2015); doi: 10.1063/1.4922856

View online: http://dx.doi.org/10.1063/1.4922856

View Table of Contents: http://scitation.aip.org/content/aip/journal/rsi/86/6?ver=pdfcov

Published by the AIP Publishing

\section{Articles you may be interested in}

Lyman- $\alpha$ radiation of a metastable hydrogen beam to measure electric fields

Appl. Phys. Lett. 99, 181502 (2011); 10.1063/1.3658459

Measurements of Local Electric Fields in Low Density Plasmas via Stark-Splitting of Hydrogen Resonance Lines

AIP Conf. Proc. 645, 86 (2002); 10.1063/1.1525440

Modeling of Doppler-Broadened Ha and Da Spectra Including Fine Structure for Moderate Static Electric

Fields

AIP Conf. Proc. 645, 79 (2002); 10.1063/1.1525439

Spherical field meter to measure the electric field vector-measurements in fair weather and inside a dust devil

Rev. Sci. Instrum. 70, 2140 (1999); 10.1063/1.1149727

Polarized laser-induced fluorescence spectroscopy for measuring electric field distribution in plasmas

Rev. Sci. Instrum. 70, 903 (1999); 10.1063/1.1149447

Frustrated by

old technology?

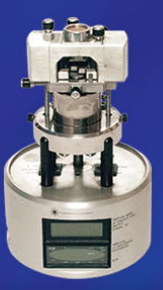

Is your AFM dead

and can't be repaired?

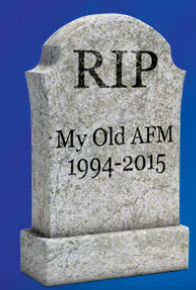

Sick of bad customer support?

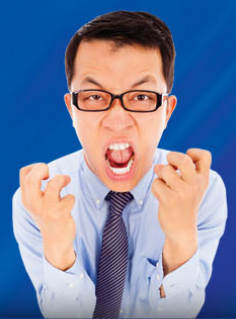

It is time to upgrade your AFM

Minimum \$20,000 trade-in discount

for purchases before August 31st

Asylum Research is today's technology leader in AFM 


\title{
Electric field induced Lyman- $\alpha$ emission of a hydrogen beam for electric field measurements
}

\author{
L. Chérigier-Kovacic, ${ }^{1, a)}$ P. Ström, ${ }^{2, b)}$ A. Lejeune, ${ }^{3, c)}$ and F. Doveil ${ }^{1, d)}$ \\ ${ }^{1}$ Aix-Marseille Université, CNRS, PIIM UMR 7345, FR-13397 Marseille Cedex 20, France \\ ${ }^{2}$ Department of Fusion Plasma Physics, Royal Institute of Technology (KTH), Teknikringen 31, \\ 10044 Stockholm, Sweden \\ ${ }^{3}$ CAMECA SAS - 29 Quai des Grésillons, 92622 Gennevilliers Cedex, France
}

(Received 19 March 2015; accepted 8 June 2015; published online 26 June 2015)

\begin{abstract}
Electric field induced Lyman- $\alpha$ emission is a new way of measuring weak electric fields in vacuum and in a plasma. It is based on the emission of Lyman- $\alpha$ radiation $(121.6 \mathrm{~nm})$ by a low-energy metastable $\mathrm{H}$ atom beam due to Stark-quenching of the $2 \mathrm{~s}$ level induced by the field. In this paper, we describe the technique in detail. Test measurements have been performed in vacuum between two plates polarized at a controlled voltage. The intensity of emitted radiation, proportional to the square of the field modulus, has been recorded by a lock-in technique, which gives an excellent signal to noise ratio. These measurements provide an in situ calibration that can be used to obtain the absolute value of the electric field. A diagnostic of this type can help to address a long standing challenge in plasma physics, namely, the problem of measuring electric fields without disturbing the equilibrium of the system that is being studied. (C) 2015 AIP Publishing LLC. [http://dx.doi.org/10.1063/1.4922856]
\end{abstract}

\section{INTRODUCTION}

Electric fields play a key role in plasmas: they interact with charged particles and are responsible for drifts and optical emission. Non intrusively measuring them, however, has been a long-standing challenge. Many experimental methods have been developed. For example, in the plasma core of tokamaks, where no material probe can be inserted, a heavy singly charged ion beam is accelerated and injected into the plasma. The interaction between the beam and the plasma electrons creates doubly charged ions which are more deflected by the magnetic field. Precisely measuring the energy of these ions gives an information on the plasma potential at the ionisation point, thus, on the electric field. ${ }^{1}$ In space, double probes are used. $^{2}$ These are extreme plasma conditions, but the problem is not really easier to solve in discharge plasmas.

Experimental study of sheaths or plasma instabilities is among the strongest motivations for electric field measurement in discharge plasmas. Sheaths are regions surrounding the wall of the plasma chamber or any "object" inserted inside a plasma, where a potential gradient is established. In plasma processing devices, this is where ions are accelerated. Plasma instabilities are responsible for energy confinement degradation or the damaging of plasma facing components. Several techniques to measure electric fields can be cited as follows:

- electrostatic probes are still widely used, ${ }^{3}$ but the significant perturbation they cause to the plasma makes the data interpretation difficult;

\footnotetext{
a)Electronic mail: laurence.kovacic@univ-amu.fr

b)Electronic mail: pestro@kth.se

c)Electronic mail: aurelien.lejeune@ametek.com

d)Electronic mail: fabrice.doveil@univ-amu.fr
}

- fluorescence-dip spectroscopy ${ }^{4}$ or laser Stark spectroscopy $^{5}$ is based on the well-known effect of an external electric field on the fluorescence spectra;

- the effect of the field on the motion of charged particles can be recorded using laser induced fluorescence (LIF) techniques. Ions can absorb a laser radiation if its frequency is resonant with their velocity (Doppler effect). When the laser frequency is varied, one obtains the velocity distribution function of the plasma ions. The electric potential in the plasma is derived from the shift of the velocity distribution function caused by the ion acceleration in the field; ${ }^{6}$

- tracking particles optically tagged by a laser with a fixed frequency ${ }^{7}$ gives the diffusion coefficient and the electric field by studying the time-dependence of the fluorescence signal during a density perturbation (the propagation of a shock wave, for example);

- using dust particles levitating above the powered electrode in a radio-frequency discharge as electrostatic probes $^{8}$ is another simple technique. It only applies when dust particles are present in the discharge, thus remaining very specific.

This list is not comprehensive. Optical methods have been developed because they are less intrusive than probes. Except laser Stark spectroscopy, these methods cannot be considered as direct. They all need elaborate and rather expensive tools, except the last one but it is very specific to one kind of study. So, there is still need for a more reliable diagnostic, where the electric field amplitude can be directly derived from the measured physical quantity. We show here a possible way using Electric Field Induced Lyman- $\alpha$ Emission (EFILE) of a probing metastable hydrogen beam. No sophisticated equipment is required. Thanks to the quantum properties of hydrogen, EFILE is sensitive to electric fields as low as 
$0.1 \mathrm{~V} / \mathrm{cm}$, and the lock-in technology allows for a very good signal to noise ratio.

This paper is focused on the extensive description of the experimental setup used to measure electric fields; from the plasma source, creating the beam to the test measurements first in vacuum, later on in a plasma, to demonstrate the feasibility of the concept. In Sec. II, we make a brief recall of the theory to explain how emission of Lyman- $\alpha$ radiation occurs when a hydrogen atom lies within an electric field and how the modulus of the field can be inferred from the line intensity. The small magnetic multi-cusp source which provides the necessary ion beam as well as the methods for producing $\mathrm{H}$ atoms in the metastable $2 s$ state and measuring the Lyman$\alpha$ intensity is presented in Sec. III. Then, we show a detailed study of the beam (Sec. IV). We have made measurements with both an ion beam and an atomic beam, both cases are discussed in Sec. V. The paper closes with a summary and perspectives.

\section{PHYSICAL PRINCIPLES}

The EFILE diagnostic is based on the Stark-mixing occurring between the levels $2 s_{1 / 2}$ and $2 p_{1 / 2}$ when a hydrogen beam is subjected to an external time-dependent or constant electric field. Although Dirac theory states that levels with the same $n$ and $j$ quantum numbers ought to be degenerate, Lamb and Retherford experimentally demonstrated that in hydrogen, the $2 s_{1 / 2}$ energy level lies above the $2 p_{1 / 2}$ level by an amount of about $\Delta E \sim 4.4 \times 10^{-6} \mathrm{eV} .{ }^{9}$ Indeed, the interaction between the electron and the quantized electromagnetic field in vacuum leads to a quantum electrodynamic effect which is responsible for the small energy shift of these levels, called Lamb-shift. Hyperfine splitting introduces additional separation, on the order of $1-5 \times 10^{-7} \mathrm{eV}$, between $2 \mathrm{p}$ substates with different total angular momentum (for the system electron plus nucleus). The $2 s_{1 / 2}$ level is metastable (lifetime of about $0.14 \mathrm{~s}$ ). Transitions to the ground state $1 s_{1 / 2}$ are forbidden; however, a quenching of the metastable state can occur if the atoms are sent through a region where an electric field is applied. Stark mixing of the $2 s$ and $2 p$ levels then results in decay to the ground state with the accompanying emission of a Lyman- $\alpha$ radiation. The timedependent Schrödinger equation is solved using perturbation theory for electric fields lower than $475 \mathrm{~V} / \mathrm{cm}$ (the limit when the Stark shift is of the same order of magnitude as the Lamb shift) in order to calculate the quenched $2 s$ level decay rate $\gamma_{2 s}^{*}$ as a function of field frequency $\omega / 2 \pi$ and magnitude $E$. As long as $E$ is lower than $E_{0}=260 \mathrm{~V} / \mathrm{cm}$ in the case of a static perturbing field or $E_{\omega}=20 \mathrm{~V} / \mathrm{cm}$ for a resonant field, the decay rate is time-independent and the calculation can be done analytically. ${ }^{10-12}$ Above the threshold field strength, the solution has to be derived numerically and exhibits saturation. This phenomenon is not visible in our data because of a much more efficient experimental saturation process, as we shall see in the following.

Calling $\omega / 2 \pi$ the frequency of the field, $\gamma_{2 p}=1 / \tau_{2 p}$ the decay rate of the $2 p$ state, $\hbar \omega_{L}$ the energy difference associated to the Lamb-shift ( $\left.v_{L}=1057 \mathrm{MHz}\right), e$ the elementary charge, and $a_{0}$ the Bohr radius, one gets the quenched $2 s$ level decay rate,

$$
\gamma_{2 s}^{*}(\omega)=\frac{9 e^{2} a_{0}^{2} E^{2}}{\hbar^{2}} \frac{\gamma_{2 p}}{\left(\omega-\omega_{L}\right)^{2}+\gamma_{2 p}^{2} / 4} .
$$

We have left out the contribution of hyperfine splitting here, but it can easily be included as done in Ref. 10. In the limit of a constant field, when $\omega \rightarrow 0$, the result of Lamb ${ }^{13}$ is retrieved. When $\omega$ approaches $\omega_{L}$, Eq. (1) features a strong resonance with an expected amplification factor of about 450, which is independent of the value of the field. We experimentally observe a factor of this order of magnitude between the signal measured when the electric field is resonant with the Lamb shift frequency and when the electric field is constant. ${ }^{14}$

Calculating the quenched level $2 s$ lifetime is a major issue since the probe $\mathrm{H}(2 s)$ beam will start to interact with the perturbing field as soon as it is sent through the explored medium. One has to be sure that the probe beam is not totally quenched before it reaches the region of interest. In all cases, the signal intensity is proportional to the square of the electric field modulus. This accounts for the theoretical ability to detect small constant fields as low as $0.1 \mathrm{~V} / \mathrm{cm}$ and even lower when the field frequency approaches the Lamb-shift frequency. The actual experimental resolution will depend on the beam current and the detection system.

\section{FROM THE HYDROGEN PLASMA TO THE LYMAN- $\alpha$ SIGNAL}

Fig. 1 shows the global experimental setup. We will describe every part of it in Secs. III A and III B. As first described by Limpaecher and MacKenzie ${ }^{15}$ in 1973, a multipolar magnetic field structure provides a high density steady state plasma by repelling the ionizing electrons towards the center of the discharge. Later on, this field structure was proven to be a very effective way to produce hydrogen ion beams with high proton ratio for neutral beam injection heating. ${ }^{16} \mathrm{We}$ had a good experience in this kind of efficient and affordable plasma source ${ }^{17,18}$ that is the reason why it has been chosen as the ion source for the development of this diagnostic. A noteworthy property of the present device, inspired by Leung et al. ${ }^{19}$ is its small size $(16.6 \mathrm{~cm}$ long and $10 \mathrm{~cm}$ in diameter), which is made possible because the only aim is to

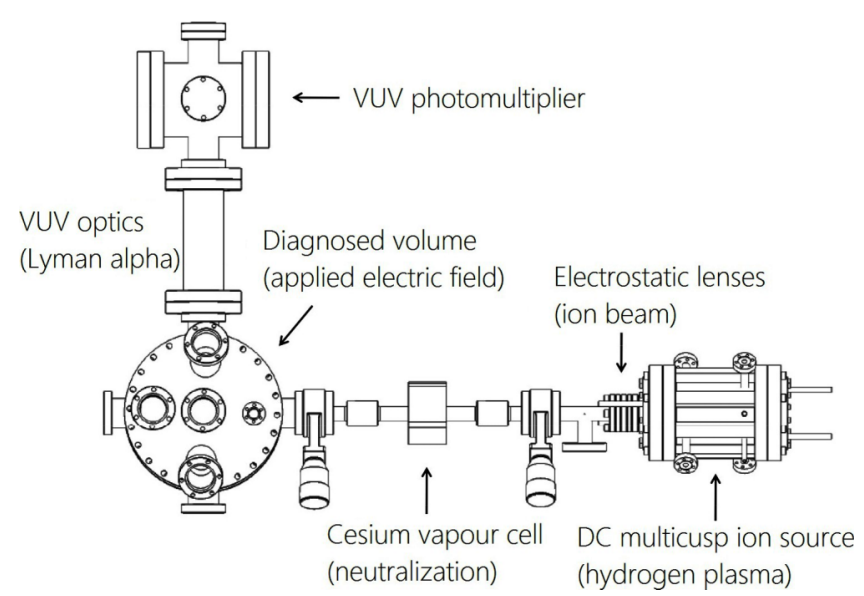

FIG. 1. Top view of the experimental device. 
(DC multicusp ion source)

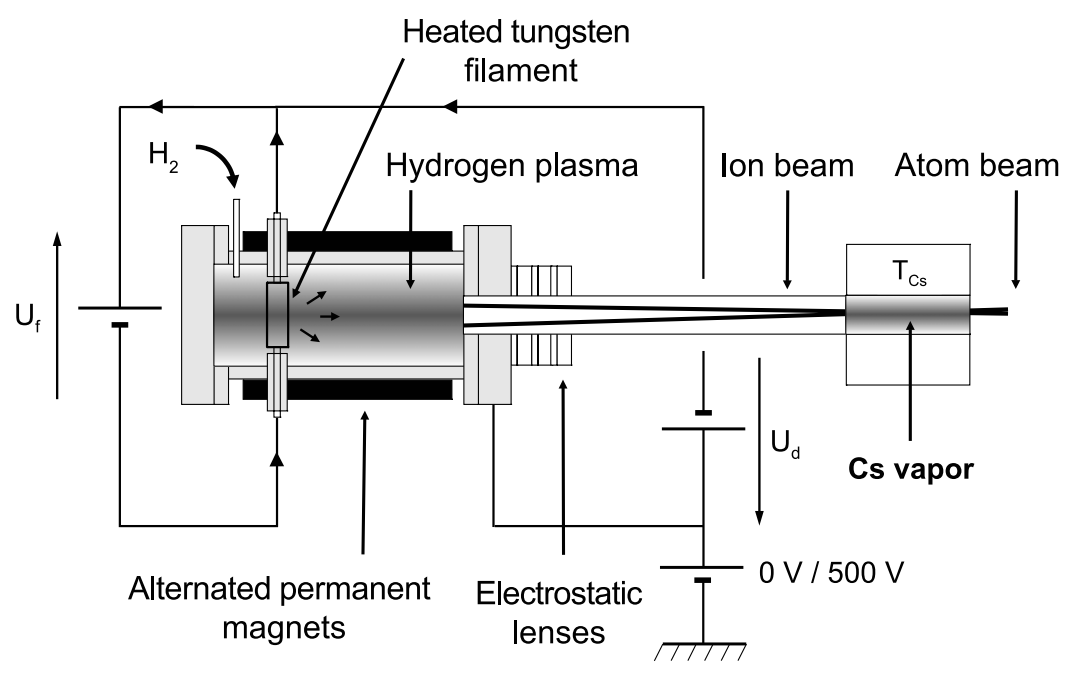

(Cs vapor cell)

FIG. 2. The plasma source. produce a low energy (between $100 \mathrm{eV}$ and $500 \mathrm{eV}$ ) ion beam. Measurements are performed inside a test chamber where that beam is focused. Many aspects of the type of discharge here described have already been treated extensively in the papers referred above. In the following, we will therefore only focus on the characteristics of our source.

\section{A. The hydrogen ion source}

A schematic view of the plasma source is displayed in Fig. 2. The magnets surrounding the vacuum vessel produce a field of $500 \mathrm{G}$ at the wall, decreasing very fast to the center. The non-magnetized volume is estimated to be about $100 \mathrm{~cm}^{3}$. Hydrogen $\left(\mathrm{H}_{2}\right)$, whose flow is controlled by a micro leak valve to maintain a low pressure in the range of $10^{-5}$ to $10^{-4} \mathrm{mbar}$, is used as working gas. Primary electrons are emitted from a W-shaped tungsten filament (cathode), heated by a $15 \mathrm{~A}$ current. Their energy is set by a discharge potential of $80 \mathrm{~V}$ applied between the filament and the walls (anode). This yields a discharge current up to $3 \mathrm{~A}$ and a mean plasma density, as it has been measured with a conventional Langmuir probe, of $10^{10} \mathrm{~cm}^{-3}$.

The plasma contains different charged species such as $\mathrm{H}^{+}$, $\mathrm{H}_{2}^{+}, \mathrm{H}_{3}^{+}$, and $\mathrm{H}^{-}$. Positive ions are extracted from the source by a focusing Einzel lens which has been designed using standard charged particles optics simulation software (SIMION ${ }^{20}$ ). The Einzel lens consists of three stainless steel electrodes separated by ceramics. Going from the source, the first electrode is biased to a positive potential and the second one to a strongly negative potential $(2 \mathrm{kV})$ to repel electrons. The potential difference between the third electrode which is grounded and the wall of the source chamber can be varied between $0 \mathrm{~V}$ and $500 \mathrm{~V}$ and sets the energy of the ions. The beam is then passed through a cesium (Cs) cell ${ }^{21,22}$ which can be heated to produce $\mathrm{Cs}$ vapor to create metastable atoms in the $2 \mathrm{~s}$ state by a charge exchange collision ${ }^{23} \mathrm{H}^{+}+\mathrm{Cs} \longrightarrow \mathrm{H}(2 \mathrm{~s})+\mathrm{Cs}^{+}$with a maximum cross section at $500 \mathrm{eV}$. If the cesium cell is not heated, the Lyman- $\alpha$ emission is significantly lower ${ }^{14}$ but the diagnostic is still working well, as metastable atoms are being produced by collisions between ions and the residual gas along the path to the test chamber. This case will be referred in the following as ion beam measurements. Measurements done with protons converted into $\mathrm{H}(2 s)$ within the cesium cell will be referred as atom beam measurements, although it should be kept in mind that heavier hydrogen charged species are not eliminated from the beam.

\section{B. The test chamber and the detection optics}

As shown in Fig. 1, after passing through the Cs cell, the beam is sent into a measurement (test) chamber. Inside the test chamber, different devices can be set up, such as an energy analyzer, or a mass spectrometer to characterize the beam, and a two-plates assembly to create a variable electric field. The upper plate is grounded and a controlled voltage is applied to the lower plate which is $5 \mathrm{~cm}$ below. Both plates are covered by a ceramic coating, only exposing a rectangular area of $1.5 \times 8 \mathrm{~cm}$ at the center. This was made with the intent of trying to make the electric field more homogenous and localized in space than with bare plates. A typical map of the potential calculated with a finite element program $\mathrm{FEMM}^{24}$ is shown in Fig. 3. This is a planar view from the side where the plates have been simply represented by two rectangular conductors $(1.5 \times 0.5 \mathrm{~cm})$, the upper one being grounded and the lower one polarized at $300 \mathrm{~V}$. The beam is running along the horizontal black line and the circle represents the viewing volume of the photomultiplier collecting the Lyman- $\alpha$ radiation. The detector used to measure the beam current and profile (white rectangle in Fig. 3) is grounded. We see that the electric field is rather homogeneous within this volume but also extends to a few millimeters outside. Indeed, we experimentally show that part of the atoms decay before reaching the diagnosed volume, leading to a saturation of the Lyman- $\alpha$ signal at high voltages. As it will be described in Sec. V B, a fit of the data gives an estimate of the length of the region where this effect takes place.

The Lyman- $\alpha$ emission at $121.6 \mathrm{~nm}$ between the plates is collected in a direction perpendicular to the beam. $\mathrm{A} \mathrm{MgF}_{2}$ 


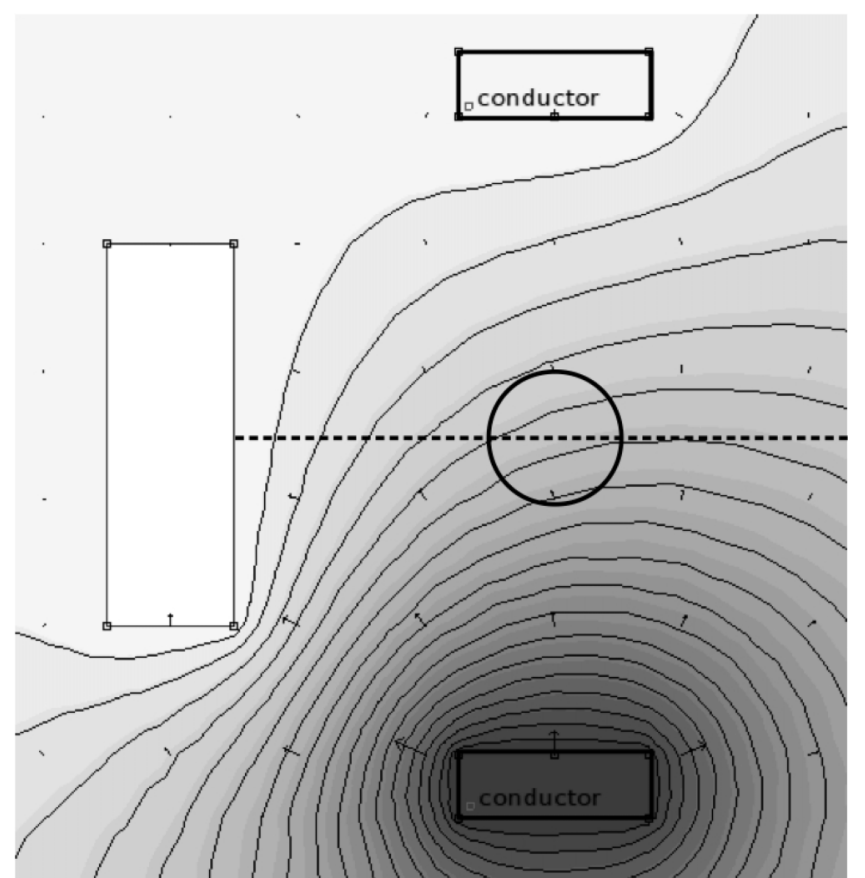

FIG. 3. Example of static potential and electric field map as calculated by FEMM: the potential values are represented by levels of gray ranging from 0 $\mathrm{V}$ (lightest) to $300 \mathrm{~V}$ (darkest); the beam path is along the horizontal line; the circle represents the diagnosed volume; and the white rectangle represents the detector used to measure the beam current and profile.

window seals the chamber containing a solar-blind UVphotomultiplier operating in vacuum. The characteristics of the photomultiplier tube are dark current $3 \times 10^{-10} \mathrm{~A}$, amplification $6 \times 10^{5}$, high voltage supply $800 \mathrm{~V}$, and cathode radiant sensitivity $28 \mathrm{~mA} / \mathrm{W}$ at $121 \mathrm{~nm}$. The diagnosed volume is imaged on the photocathode by a lithium fluoride $(\mathrm{LiF})$ lens with a magnification equal to 1 in order to get the maximum of signal in this development phase. The transparency of the window+lens assembly is estimated to $36 \%$. Spatial resolution is only determined by the size of the photocathode, and the diagnosed volume is thus approximately $1 \mathrm{~cm}$ long.

In order to improve the signal to noise ratio, a lock-in amplifier technique is used. Basically, the photomultiplier signal is multiplied by a low-frequency reference signal and a low-pass filter allows to retrieve its amplitude. The timeconstant is set between 3 and $10 \mathrm{~s}$ and the lock-in has an input impedance of $100 \mathrm{M} \Omega$ in voltage input mode. When the nongrounded plate is biased at a frequency of typically $1 \mathrm{kHz}$, the signal is the mean response to an alternately positive and negative amplitude modulation. The continuous background of spontaneous emission from the beam is not recorded. Only the part of the signal due to the field, synchronized with the biased plate modulation, is observed. Except in cases where the electric field can be controlled externally, this is however not how the diagnostic should be operated for most future applications, where the electric field is supposed to be unknown. The alternative is then to pulse the beam by means of the 500 $\mathrm{V}$ bias applied to the source. Contrary to the pulsed platevoltage mode, this does not eliminate the background signal due to spontaneous emission, which has to be subtracted after a measurement without electric field.

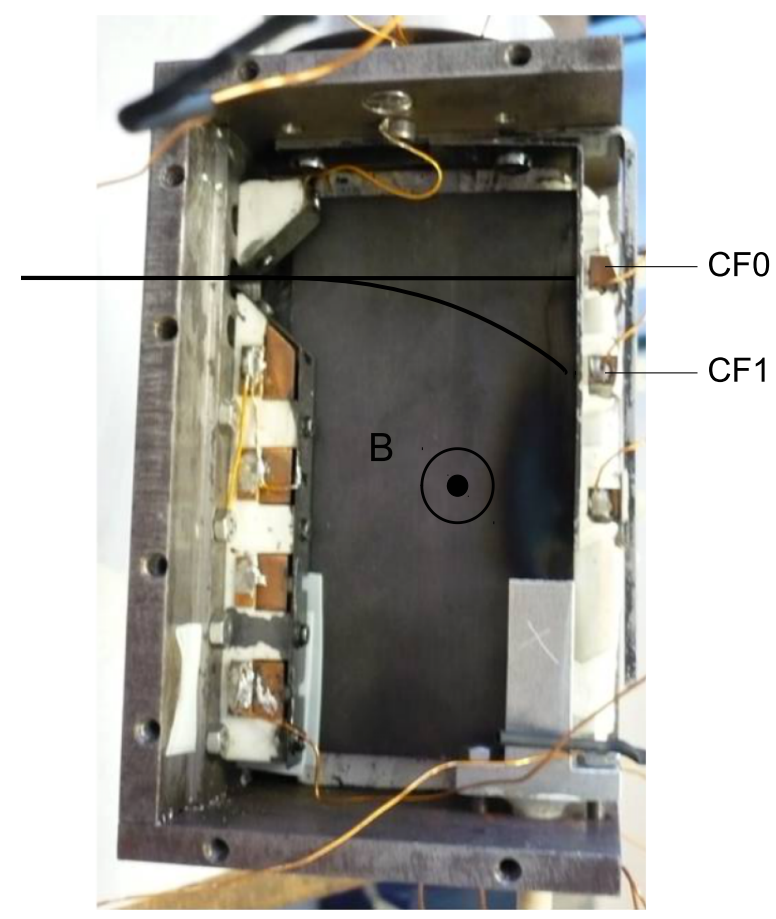

FIG. 4. Photo of the mass spectrometer used to measure $\mathrm{H}^{+}, \mathrm{H}_{2}^{+}$, and $\mathrm{H}_{3}^{+}$ density.

\section{STUDY OF THE ION BEAM}

Population ratios within the extracted beam have been measured by means of a custom mass spectrometer showed in Fig. 4, based on the model of Ehlers et al. ${ }^{25}$ The beam is entering the mass spectrometer through a $1 \mathrm{~mm}$-slit. It is deviated by a magnetic field produced by two coils of 260 turns where an adjustable current circulates. The field is measured by a Hall probe and can be varied from zero to a maximum of about 800 G. As shown in Fig. 4, two Faraday cups measure the beam current: CF0 gives the total current when there is no field and CF1 measures the ion current depending on the applied magnetic field. In our experimental conditions, the typical value of the total beam current is about $0.1 \mu \mathrm{A}$. The $\mathrm{H}^{+}, \mathrm{H}_{2}^{+}$and $\mathrm{H}_{3}^{+}$ions are detected at $B=320 \mathrm{G}, 450 \mathrm{G}$, and $560 \mathrm{G}$, respectively.

Ion density ratios have been measured over a wide range of the plasma discharge parameters (discharge current and voltage, filament heating current, pressure, etc.) to help in determining the best experimental conditions. Since we aim at working with metastable atoms, the highest possible proton density is required. The mass spectrometer is set up in the measurement (test) chamber about $60 \mathrm{~cm}$ away from the exit of the source. The test chamber is twice as large as the source chamber and evacuated by its own pumping device to ensure a good stability of its background pressure even when the pressure is varied in the source.

Figure 5 shows the ion density ratios within the beam as a function of the pressure in the source chamber for a discharge voltage $V_{d}=80 \mathrm{~V}$ and a mean discharge current of $1.65 \mathrm{~A}$. The fraction of $\mathrm{H}^{+}$increases very slowly in the considered pressure range, without being higher than $15 \%$. For a pressure lower than $5 \times 10^{-5}$ mbar, $\mathrm{H}_{2}^{+}$is the dominant species but at higher pressures, $\mathrm{H}_{3}^{+}$becomes the dominant species. These 


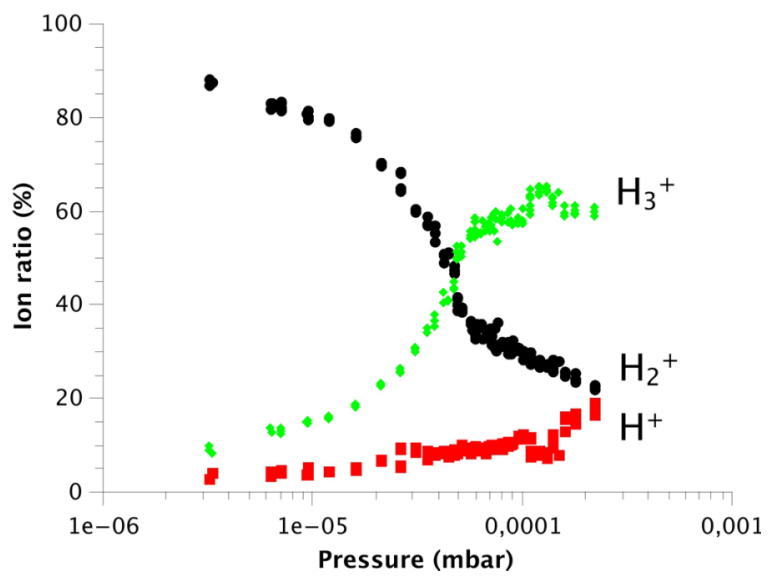

FIG. 5. $\mathrm{H}^{+}, \mathrm{H}_{2}^{+}$, and $\mathrm{H}_{3}^{+}$density ratios.

results look very much like Leung's. ${ }^{19}$ As we will discuss later, the Lyman- $\alpha$ emission from the beam in the presence of an electric field actually occurs whether the ion beam has been converted into metastable atoms in the Cs cell or not. These measurements explain why the pressure, thus the composition of the beam, has a direct influence on the Lyman- $\alpha$ signal when protons are not converted into metastable atoms in the Cs cell. On the contrary, when working with an atomic beam, we observe that the Lyman- $\alpha$ signal does not vary a lot with pressure since the $\mathrm{H}^{+}$density remains rather constant. Increasing the discharge current up to $2.5 \mathrm{~A}$ results in a better $\mathrm{H}^{+}$rate without actually being higher than $15 \%$. But the overall density of the ion beam is increasing too, providing us with a sufficient signal level.

Fig. 6 shows the Lyman- $\alpha$ intensity (solid circle) induced by a field of $23.5 \mathrm{~V} / \mathrm{cm}$ as a function of the cesium cell temperature, along with the decreasing ion current (open square) measured on the CF0 detector of the mass spectrometer. The maximum intensity is obtained for a temperature of $120{ }^{\circ} \mathrm{C}$ whatever be the parameters of the discharge. For a temperature above $120{ }^{\circ} \mathrm{C}$, the current becomes negative, indicating the presence of $\mathrm{H}^{-}$ions. These ions are also known to be produced by interaction between $\mathrm{H}^{+}$and cesium. ${ }^{23}$

The ion beam profile has also been recorded, by moving a detector vertically across the beam after the plates assembly.

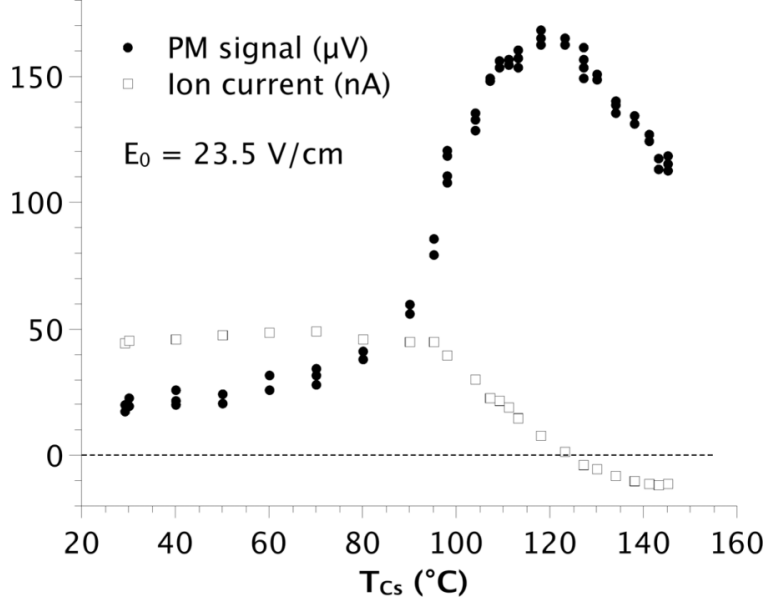

FIG. 6. Measurements as a function of the Cs temperature.

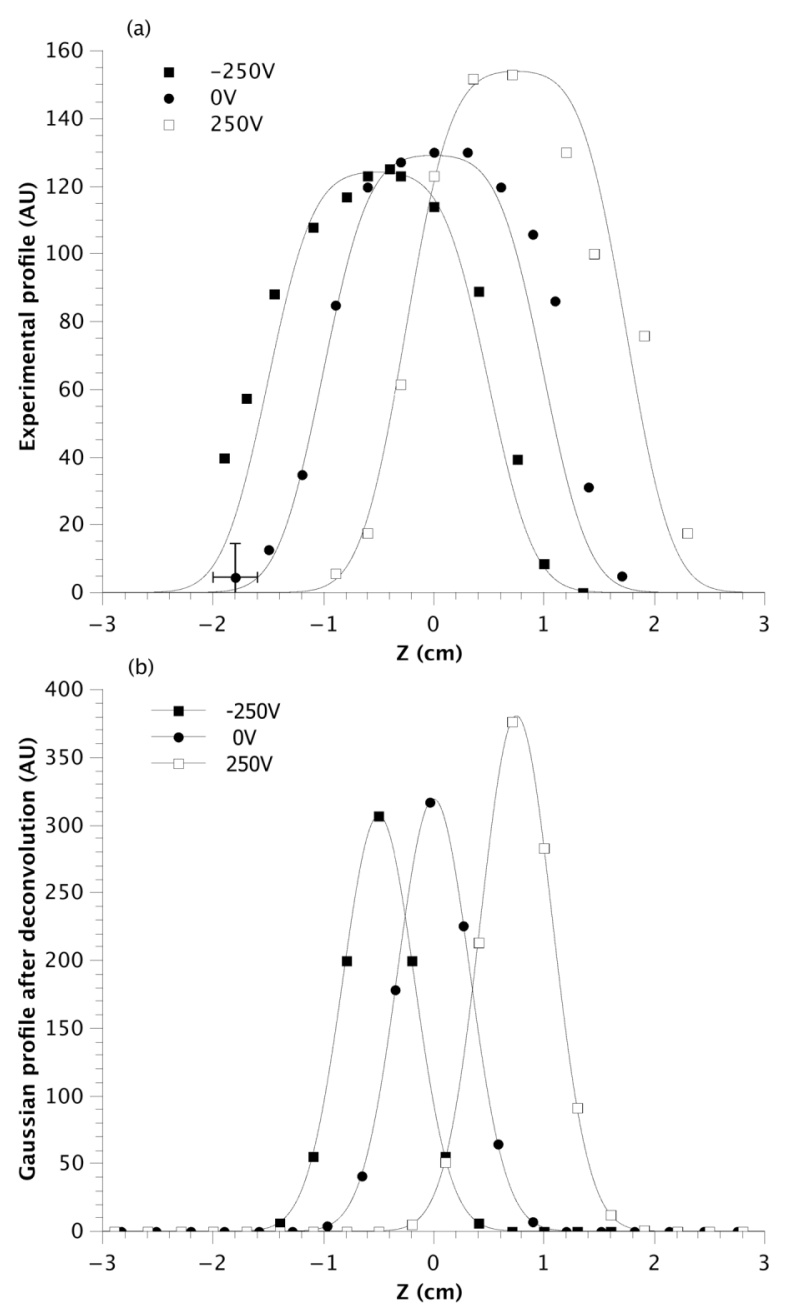

FIG. 7. (a) Experimental measurement of the beam profile. (b) Beam profile after deconvolution.

Results are displayed in Fig. 7(a), where symbols represent experimental data, and lines are a fit of the data by a Gaussian profile (shown in Fig. 7(b)) convoluted by a $1 \mathrm{~cm}$-wide boxcar function representing the instrument function of the analyzer. When no electric field is applied, the beam is centered (black circle). Plates are located at $\pm 2.5 \mathrm{~cm}$, the polarized plate being on the negative side. When a $\pm 250 \mathrm{~V}$ is applied to the lower plate, we observe a $\pm 1 \mathrm{~cm}$ deviation of the positive beam according to the direction of the field, as well as a small increase of the signal for positive biasing, when the beam is repelled from the polarized plate. We conclude from this that the beam full width at half maximum is approximately $1.3 \mathrm{~cm}$ at this point. This can depend on the Einzel lens voltage which, in our case, is adjusted for a maximum signal intensity.

\section{MEASUREMENT OF AN ELECTRIC FIELD IN VACUUM}

\section{A. E-square dependence}

First, we estimate the expected signal considering a test volume $\mathcal{V}$ of length $L=1 \mathrm{~cm}$ crossed by a beam of density $n_{b}$ and velocity $v$ so that the particle flux density is $J=n_{b} v$. 
The fraction of particles which decay during transit through $\mathcal{V}$ is $f_{\mathcal{V}}=1-\exp \left(-t_{L} / \tau_{2 s}^{*}\right)$ where $t_{L}=L / v$ is the residence time of a particle in $\mathcal{V}$. But as it is shown by Fig. 3, the electric field extends to each side of the test volume. Consequently, only a fraction $f_{0}$ of particles survive to reach the field of view of the photomultiplier, after having experienced a field along a mean distance of $z_{0}$ before arriving to the test volume: $f_{0}$ $=\exp \left(-z_{0} /\left(\tau_{2 s}^{*} v\right)\right)$. The signal amplitude is then proportional to $f_{0}$ multiplied by the fraction $f_{\mathcal{V}}$ of decaying atoms in the volume $\mathcal{V}$ and the number of decays per unit of time from the test volume is

$$
I_{\alpha}=f_{0} f_{\mathcal{V}} J \mathcal{V} / L \propto\left(1-\exp \left(-L /\left(\tau_{2 s}^{*} v\right)\right)\right) \exp \left(-z_{0} /\left(\tau_{2 s}^{*} v\right)\right) .
$$

As long as the residence time $t_{L}\left(\approx 10^{-8} \mathrm{~s}\right.$ in our case $)$ is short in comparison with the lifetime $\tau_{2 s}^{*}$, this condition being satisfied for static electric fields below $65 \mathrm{~V} / \mathrm{cm}$ with a 500 eV beam, Eq. (2) can be simplified and gives $I_{\alpha}=n_{b} \mathcal{V} \gamma_{2 s}^{*}$. The signal $S$ measured by the photomultiplier is equal to $I_{\alpha}$, multiplied by the fraction $\Omega$ of the radiation, intercepted by the detector, and converted into a signal,

$$
S=K \Omega E^{2},
$$

where $E$ is the modulus of the electric field and $K$ an amplitude factor which includes the quantum efficiency and the current amplification of the photomultiplier.

As a first example, the $\mathrm{E}^{2}$ dependence indicated by Eq. (3), measured with the ion beam not converted into $\mathrm{H}(2 s)$ in the cesium cell for both pulsed-beam and pulsed-voltage modes, is displayed in Fig. 8 for an applied voltage between the plates lower than 300 V. For a better comparison, pulsed beam signal can be read on the left axis (which starts from the level of spontaneous emission) and pulsed voltage signal can be read on the right axis (which starts from zero since spontaneous emission is canceled by pulsing the electric field). A quadratic law fits both cases. The values are about 20 times higher in the pulsed voltage mode, essentially indicating that the creation process of the metastable atoms is more efficient when the beam is not pulsed.

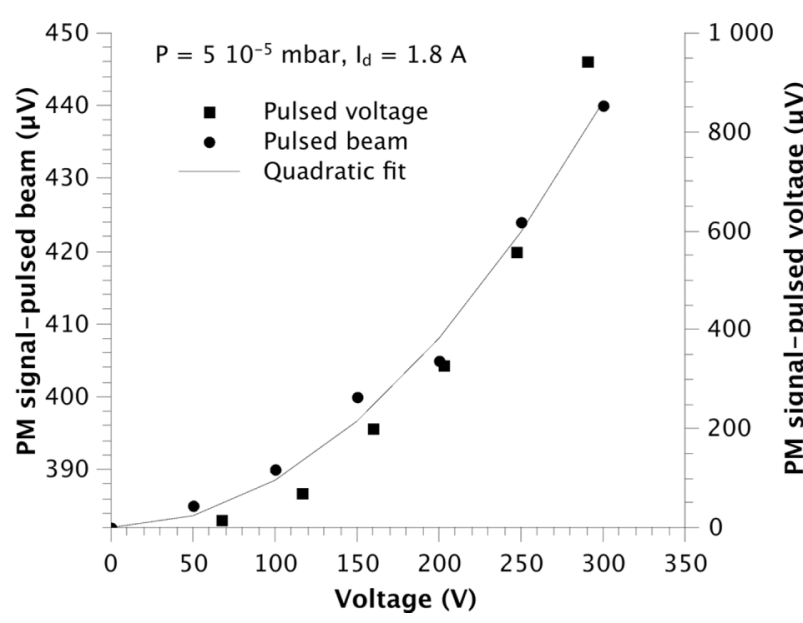

FIG. 8. Comparison between measurements with pulsed beam and pulsed plate-voltage.

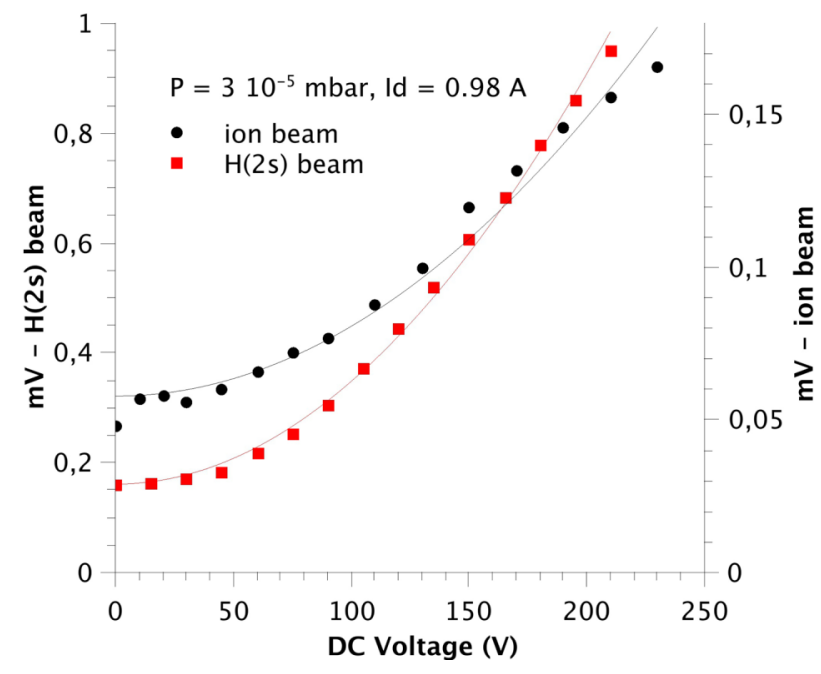

FIG. 9. Comparison between measurements with atomic and ion beam.

As a second example, Fig. 9 shows the comparison between measurements with and without conversion of protons in the cesium cell for a static applied voltage. Again, a quadratic function of the voltage fits both cases and the value of the coefficient of the parabola is about 10 times higher in the atomic beam case.

\section{B. Saturation of the signal}

Fig. 10 shows the Lyman- $\alpha$ signal vs. the voltage applied to the plate (electric field) in pulsed atomic beam mode at a pressure of $2.8 \times 10^{-5}$ mbar for a wider range of voltage than in Fig. 8 . The asymmetry between the positive and negative potential values has been observed over a wide range of pressure and energy of the beam. It is explained by the fact that there are still positive and negative ions in the beam. As we already showed in Fig. 7, these ions are deviated up or down according to the field direction, probably modifying the field itself by charging the ceramic coated parts of the plates assembly.

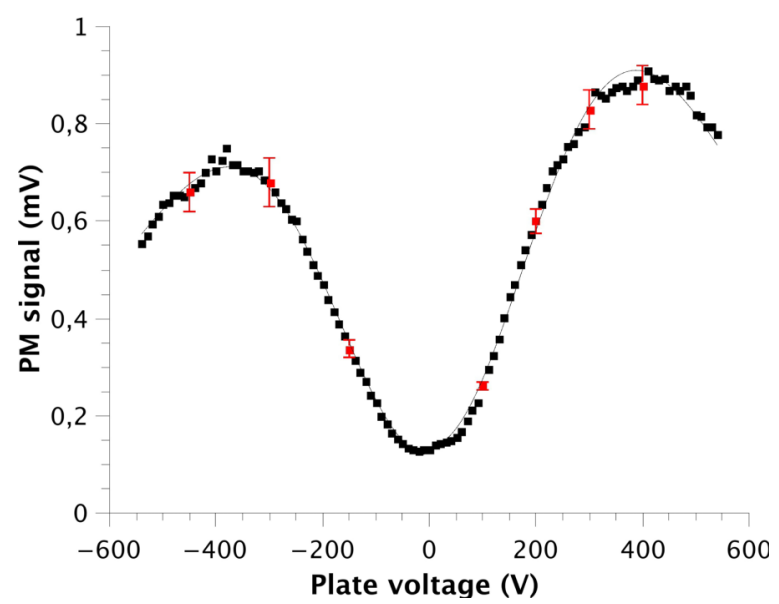

FIG. 10. Measurement of the Lyman- $\alpha$ signal as a function of the voltage applied to the plate, $500 \mathrm{eV}$ pulsed beam; continuous line is a fit by an empirical formula. 
Both sides of the curve show saturation at high field amplitudes (when the voltage is increased above approximately 350 V). As we have calculated before, for field modulus higher than $65 \mathrm{~V} / \mathrm{cm}$, Eq. (3) is no longer valid, because the lifetime $\tau_{2 s}^{*}$ is no longer negligible compared to the time of transit into the test volume. Moreover, as stated before, the beam interacts with the field before entering the diagnosed volume. This leads to a saturation which is much more efficient than the numerically predicted saturation mechanism. The curve even exhibits a decrease of the signal for higher voltages, when all metastable atoms are likely to be quenched before the region viewed by the photomultiplier.

Using Eq. (2) and taking into account the spontaneous emission from $2 p$ by adding a constant $\mathrm{B}$, we fit the data with a function of the form

$$
S(V)=A \exp \left(-C_{0} V^{2}\right)\left(1-\exp \left(-C_{L} V^{2}\right)\right)+B,
$$

where $V$ is the plate voltage. This curve will be referred as the calibration curve for the next measurement. It is noticeable that two parameters in this expression do not have to be varied: $B$ is directly taken from the signal at zero field and $C_{L}$ is fixed by $L \approx 1 \mathrm{~cm}$, the width of the diagnosed volume. The parameter $C_{0}$ gives the length of the undetected decay region $z_{0}$, which is about $2.5 \mathrm{~mm} \pm 0.5 \times 10^{-2} \mathrm{~mm}$ for the positive branch and $2.9 \mathrm{~mm} \pm 0.5 \times 10^{-2} \mathrm{~mm}$ for the negative one. This difference builds upon the already stated fact that charge accumulation effects give rise to asymmetries. It is concluded that it does not only change the field strength but also the geometry of the field. This indicates clearly that the original intent of adding a coating, namely, to localize the electric field to the measurement region, is not fulfilled. The order of magnitude of $z_{0}$, however, is consistent with what is observed in Fig. 3. Regarding the calibration, the same discharge parameters in the source will produce the same ion beam. The ion conversion in the cesium oven keeps the same efficiency over years (only cesium lost by accident made us reload cesium in the oven). Calibration curves are fairly reproducible, the parameters of the fit $\mathrm{A}$ and $\mathrm{C} 0$ depending only on discharge parameters. Thus, calibration does not need to be performed at the same time as the experiment.

\section{Electric field profile between two biased plates measured from the atomic beam}

To test the EFILE diagnostic in vacuum, we measure the Lyman- $\alpha$ line intensity as a function of the position of the beam between the plates. The detection optics being fixed, we do this by moving the plate assembly up and down. We polarize the lower plate at $+300 \mathrm{~V}$ and operate in pulsed atomic beam mode. The pressure in the source is set at $3 \times 10^{-5} \mathrm{mbar}$ and the discharge current is set at $0.95 \mathrm{~A}$. We first record the Lyman- $\alpha$ signal when no field is present (full square symbols in Fig. 11, grounded plate on the left). As expected, the signal is constant except in the vicinity of the plates, where it decreases by at least a factor of 2. In this region, the beam and the viewing volume are both screened by the plates. This screening effect is also visible on the raw Lyman- $\alpha$ signal (full circle symbols in Fig. 11) in the presence of the field, although we shall prove

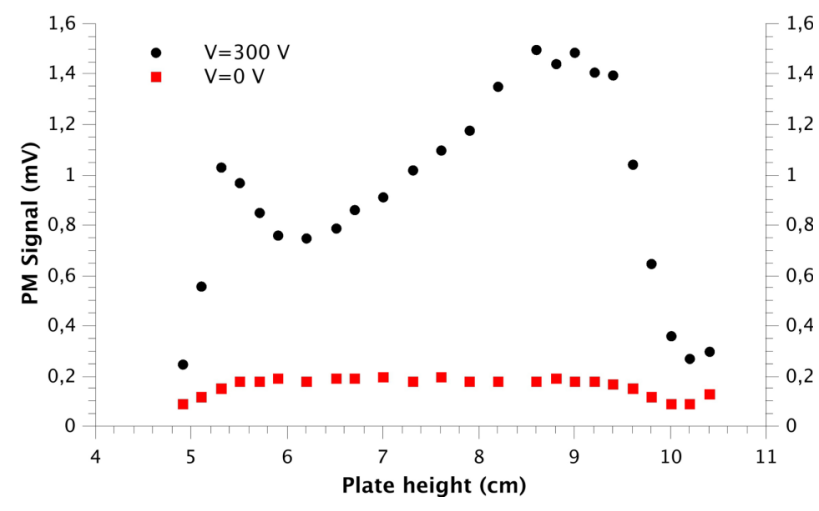

FIG. 11. Measurements of the electric field profile between the plates in vacuum: raw data.

that it is not the only reason why the signal is decreasing near the plates.

As we have seen in Figs. 8 and 9 and from the theory, the intensity of the Lyman- $\alpha$ line measured at the center of the plate assembly is proportional to the square of the modulus of the field, as long as the plate voltage remains below approximately $300 \mathrm{~V}$. However, this does not stand near the polarized plate, where the expected electric field rises, as indicated by the tightening of the equipotential lines (Fig. 3). Actually, the signal is saturated because the field value is higher than the value at the maximum of the calibration curve (Fig. 10). We thus use the empirical curve in Fig. 10 to retrieve the field values of Fig. 12. Basically, for each value of the Lyman- $\alpha$ signal, we can find two corresponding values of the field strength. Between the left (grounded) plate and the position of the maximum of the raw curve, we consider that the signal is not saturated and the corresponding value ought to be found on the left side of the maximum of the calibration curve (for positive values of the potential), the field amplitude being obtained by dividing the potential by the distance between the plates. On the contrary, near the polarized electrode, by considering a saturated value of the Lyman- $\alpha$ signal, we use the value on the right side of the maximum of calibration curve.

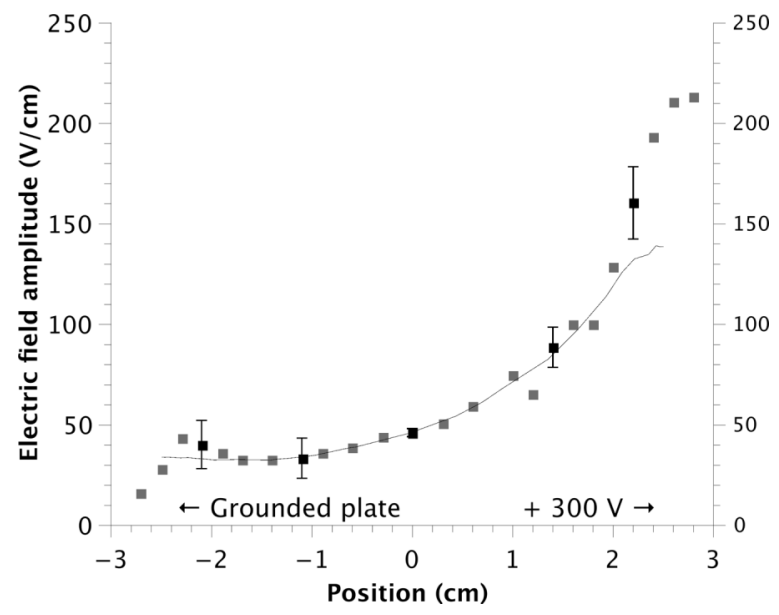

FIG. 12. Comparison between the reconstruction of the electric field profile between the plates in vacuum (square) and the calculation by a finite element method (solid line). 
Fig. 12 shows that the FEMM calculation (solid line) is in good agreement with the electric field profile reconstructed from the experimental data, in particular at the center of the device. Error bars are derived from the error bars in Fig. 10: the uncertainty on the PM signal gives the corresponding interval of error on the field amplitude. We find that the electric field near the polarized plate increases much more than the FEMM calculation. Although these measurements were done with the atomic beam, this is likely to be a consequence of the positive and/or negative charges still present in the beam, which are charging the ceramic coated part of the plates, as already stated before. Measurements at different voltages show more or less the same effect, whether the charged particles are less or more deviated by the field. This effect is even already visible on raw data at zero field when measurements are done with the ion beam. This will be the aim of further investigations.

\section{CONCLUSION}

EFILE is a new method for measuring the electric field modulus. Measurements can be made directly with a hydrogen ion beam or with an atomic beam after a charge exchange process with cesium. We prove that the results are perfectly consistent and exploitable. We experimentally find the quadratic behavior of the Lyman- $\alpha$ intensity at low electric field amplitude. When the voltage applied between two plates is increased, we explain the saturation of the signal by the loss of atoms decaying in a short region before the diagnosed volume. An estimate of the size of this region is given by a fit of the data with a two-parameter model. The observed positive/negative voltage asymmetry is just a specific feature of our experiment: the charging of the ceramic coating of the plates changes the geometry of the field, as is shown by the electric field profile measurement between the plates. Indeed, this profile is in accordance with the result of a finite element method calculation, except in the vicinity of the plates because of the charge accumulation on the ceramic coating. The EFILE diagnostic can readily be extended to plasma sheath measurements: ${ }^{26}$ this work is still in progress. This diagnostic proves to be very useful and efficient for measuring electric field values as low as $1 \mathrm{~V} / \mathrm{cm}$ in vacuum and plasmas.

\section{ACKNOWLEDGMENTS}

The authors are grateful to E. Garabedian, J.-P. Busso, A. Totin, and B. Squizzaro for technical assistance. They acknowledge the help of G. Bachet and G. Prasad for the conception and construction of the experimental setup. P.S. benefited from an Erasmus exchange grant via the European Lifelong Learning Programme 2007-2013. A.L. benefited from a grant by Ministère de la Recherche.

${ }^{1}$ X. Z. Yang et al., Phys. Fluids B 3, 3448 (1991).

${ }^{2}$ S. Ratynskaia, V. Demidov, and K. Rypdal, Phys. Plasmas 9, 4135 (2002).

${ }^{3}$ B. Vara, C. S. Dalal, S. Karkari, and H. Kabariya, IJERA 4, 244 (2014), see http://www.ijera.com/papers/Vol4_issue3/Version\%201/AU4301244247. pdf.

${ }^{4}$ U. Czarnetzki, D. Luggingölscher, and H. F. Döbele, Plasma Sources Sci. Technol. 8, 230 (1999).

5 J. P. Booth, M. Fadlallah, J. Derouard, and N. Sadeghi, Appl. Phys. Lett. 65, 819 (1994).

${ }^{6}$ G. Bachet, L. Chérigier, M. Carrère, and F. Doveil, Phys. Fluids B 5, 3097 (1993).

${ }^{7}$ N. Claire, M. Dindelegan, G. Bachet, and F. Skiff, Rev. Sci. Instrum. 72, $4372(2001)$

${ }^{8}$ A. A. Samarian and B. W. James, Plasma Phys. Control. Fusion 47, B629 (2005).

${ }^{9}$ W. E. Lamb and R. C. Retherford, Phys. Rev. 72, 241 (1947).

${ }^{10}$ P. Ström, M.S. thesis, Université d'Aix-Marseille, 2013, see http://urn.kb. se/resolve?urn=urn\%3Anbn\%3Ase\%3Auu\%3Adiva-206123.

${ }^{11}$ A. Lejeune, Ph.D. thesis, Université de Provence, 2010.

${ }^{12}$ J. F. Benage, Jr., Ph.D. thesis, University of Colorado, 1986.

${ }^{13}$ W. E. Lamb and R. C. Retherford, Phys. Rev. 79, 549 (1950).

${ }^{14}$ A. Lejeune, L. Chérigier-Kovacic, and F. Doveil, Appl. Phys. Lett. 99, 181502 (2011).

${ }^{15}$ R. Limpaecher and K. R. MacKenzie, Rev. Sci. Instrum. 44, 726 (1973).

${ }^{16}$ Y. Okomura, H. Horiike, and K. Mizuhashi, Rev. Sci. Instrum. 55, 1 (1984).

${ }^{17} \mathrm{G}$. Bachet, L. Chérigier, and F. Doveil, Phys. Plasmas 2, 5 (1995).

${ }^{18}$ M. Carrère, L. Chérigier, C. Arnas-Capeau, G. Bachet, and F. Doveil, Rev. Sci. Instrum. 67, 4124 (1996).

${ }^{19}$ K. N. Leung, R. D. Collier, L. B. Marshall, T. N. Gallaher, W. H. Ingham, R. E. Kribel, and G. R. Taylor, Rev. Sci. Instrum. 49, 321 (1978).

${ }^{20} \mathrm{See}$ http://www.simion.com for information about ion optics simulation.

${ }^{21}$ M. Bacal, A. Truc, H. Doucet, H. Lamain, and M. Chrétien, Nucl. Instrum. Methods 114, 407 (1974).

${ }^{22}$ M. Bacal and W. Reichelt, Rev. Sci. Instrum. 45, 769 (1974).

${ }^{23}$ P. Pradel, F. Roussel, A. Schlachter, G. Spiess, and A. Valance, Phys. Rev. A 10, 797 (1974).

${ }^{24}$ See http://www.femm.info for homepage of Finite Element Method Magnetics for solving electrostatic problems.

${ }^{25}$ K. W. Ehlers, K. N. Leung, and M. D. Williams, Rev. Sci. Instrum. 50, 1031 (1979).

${ }^{26}$ F. Doveil, A. Lejeune, and L. Chérigier-Kovacic, Phys. Plasmas 20, 055701 (2013). 